\title{
An Analysis of the Function and Application of Metaphor in Political Discourse
}

\author{
Xirong Ai \\ School of Foreign Languages, Yulin University, Yulin 719000, China \\ 353217985@qq.com
}

\begin{abstract}
Metaphor is an important form of rhetoric and an important way of human cognition. The use of metaphor in political discourse can play a role in persuading listeners, shaping audience cognition, and constructing relevant policies. Based on this, metaphor becomes one of the important policy tools that politicians rely on and use. On the basis of presenting and analyzing the relevant theories, this paper illustrates the function and application of metaphor in political discourse through the case study of climate change policy construction of Clinton administration and George W. Bush administration.
\end{abstract}

Keywords: metaphor; political discourse; function; application.

\section{隐喻在政治话语中的功能和运用分析}

\section{艾喜荣}

榆林学院外国语学院, 陕西榆林, 中国

摘 要: 隐喻是一种重要的修辞形式, 更是一种重要的人类认知方式。在政治话语中隐喻的 使用可以起到说服听众、塑造听众认知、进而建构相关政策的作用。基于此, 隐喻成为政治 家们所依赖和使用的重要政策工具之一。在对相关理论进行梳理和分析的基础上, 本文以克 林顿政府与小布什政府气候变化政策建构为案例, 对政治话语中的隐喻的功能和运用进行了 阐释。

关键词: 隐喻; 政治话语; 功能; 运用。

\section{1. 前言}

隐喻是一种重要的修辞形式, 更是一种重要的人类认知方式。隐喻是人类交际史上研究最多、 分析最多的语言手段之一。 ${ }^{[1]}$ 基于隐喻在人类认知和交际中的重要作用, 它在政治话语中得 到了频繁的使用，成为政治家们所依赖的重要政策工具之一。

首先, 从传统的修辞学角度来看, 隐喻就是用一种事物指代另一种完全不同的事物, 用一种 事情或经验理解和经历另一种事情或经验。《美国传统词典》 (The American Heritage Dictionary）把 “隐喻” 定义为 “一种修辞形式, 即通常指定一个事物的词或短语用来指定另 一个事物, 从而形成一种隐含的比较” ${ }^{[2]}$ 。具体来讲, 隐喻就是通过人类的认知和推理将一 个语义源域（semantic source domain）系统地和对应地投射映合 (projecting 或 mapping) 到另一 个语义目标域（semantic target domain），以此建立起不同概念之间的相互联系。源域通常是 那些我们作为人类可以很容易地思考的东西, 或者是我们得心应手和熟悉的物质世界部分。 而目标域通常是概念性的, 它对于我们的感官来说具有隐藏性而不为我们所知。 ${ }^{[3]}$ 总体而言, 对于日常生活世界外的事物, 我们一无所知; 而理解感官不能感知世界的唯一途径在于, 将 日常生活世界同抽象世界进行相似性转换。这方面可以借助人们熟悉的参照物, 使抽象世界 被人理解。这种将已知运用于未知的原则构成了隐喻的本质。日常生活的概念及观念被用在 抽象世界，使之成为人们已知的现象而被人们所接受。 
作为修辞学意义上的隐喻, 其使用只是为了达到特殊的修辞或交际效果, 只是一种语言的装 饰与修饰。而在现代隐喻理论看来, 隐喻不仅仅是一种语言修辞手段, 更是一种思维方式和 认知方式, “隐喻无处不在, 人类的整个概念系统都是建立在隐喻基础之上的”。隐喻 “不 仅仅是语言形式，不仅仅是认识事物的过程中以一个事物来认识另一个事物的这种潜在的隐 喻思想” ，隐喻更是人类普遍的认知工具, 是用一种事物来认识、理解、思考和表达另一种 事物的过程, 是构筑概念系统的必要手段。“隐喻可以建构我们对现实的看法, 建构我们的 思维方式, 也可以建构我们的行为方式”。 ${ }^{[5]}$ 对隐喻的研究表明隐喻在构建世界观、简化对 复杂难懂状况的描述、组织公众的特定观念方面卓有成效。 ${ }^{[6]}$ 隐喻可以为奇异和抽象的事物 或现象提供一个替代性框架, 它可以使人们借助熟悉的事物或观点来认识新奇陌生的事物或 现象, 可以说是我们认识事物和了解世界的重要工具。

\section{2. 政治话语中隐喻的作用和功能}

隐喻在政治中具有重要作用。政治领域的隐喻有着与其他如日常生活方面的隐喻相同的运作 方式, 它们有助于听者借助生活中熟悉的概念或事物来达到对社会的一种外在的、难以理解 的观念的把握。作为一种探索政治语义域中新概念的启发式方法, 隐喻可以被政治党派创造 性地利用，也被官员和其他能优先使用大众媒体的人员所使用。 ${ }^{[7]}$ 诺曼 - 费尔克拉夫 (Norman Fairclough）指出, 隐喻是政治组织、大众传媒和其他机构的社会控制工具。 ${ }^{[8]}$ 政治家通过隐 喻的使用可以更有效地表达与传递他们的观点和政策, 也能更有效地说服听众。此外, 隐喻 在建构社会与政治现实方面也具有核心作用。 ${ }^{[5]}$ 保罗 - 里克尔 (Paul Ricouer) 指出隐喻具有重 新描述现实的奇特功能。 ${ }^{[9]}$ 因此, 基于隐喻对于人们认知方面的独特而重要的影响, 政治家 在政治话语中通过隐喻的使用可以影响和建构听众对于现实的看法。例如, 在第一次海湾战 争中, 老布什总统就使用了这样一些隐喻——萨达姆是恶棍（villain）, 科威特是受害者, 美 国是英雄，以此建构美国进兵海湾地区的合法性和民众对此行动的支持与认同。

隐喻在认知方面的强大作用使得它在框定中的运用也具有独特的效果。通过隐喻表达的使用 而对抽象的概念进行的语言框定, 可以激活抽象概念的隐喻表征并对推理产生影响。例如, 隐喻性地把犯罪框定为 “一种病毒困扰着城市” 或者框定为 “一只野兽肆虐着城市” 使人们 对假设的犯罪浪潮提出了不同解决方案。 ${ }^{[10]}$ 而事实上, 隐喻本身就可以被看作是用来对问题 进行概念化的显性框架。“当我们通过一个隐喻而不是别的隐喻来表示事物, 我们就用这种 方式而不是别的方式建构我们的现实” “11]。因此, 隐喻在框定中被频繁的使用也不是偶然的。 隐喻性框定被广泛地运用于公共话语中（例如杂志社论、政治宣传广告等）, 以此来对有争 议性的社会政治问题进行交流和沟通。此外，隐喻性框定也被用作其他一些有争议性话题的 交流，如恐怖主义、移民问题、战争、民权等。大量的证据表明，隐喻性框定会迅速激发受 众迁移他们对于隐喻中的抽象概念知识，进而理解目标问题中的类比性特征。

基于隐喻在认知方面的优势, 公共话语或政治话语中的隐喻性框定可以有助于受众对于框定 者的框定意图的理解与把握, 有助于受众对于问题或政策的理解, 也是对相关话题进行沟通 与交流的有效工具。隐喻以这样一种方式对一个话题进行框定, 使得对问题的这种理解优于 其他可能的理解。 ${ }^{[13]}$ 在框定中隐喻的使用一方面可以使受众更加容易理解并接受一个框架, 另一方面也可以起到建构受众对这一框架认知的作用。隐喻性框定的影响效果可能是隐性 的：人们没有意识到隐喻对他们所作出决定的影响；相反人们依靠更加 “实质性”（常常是 数字性）的信息作为他们作出解决问题决策的动力。但是, 一个隐喻微妙的实例化（通过一 个词）可以极大地影响人们如何解决类如犯罪等社会问题以及如何收集充分的信息而做出决 定。 ${ }^{[14]}$ 正因为在人类认知中具有重要的作用, 隐喻性框定在政治实践中被大量地使用。例如, “战争” 就是一个在美国政治中经常被使用的框定性隐喻, 用以表示一些外在的、生成性的 和真正的威胁, 例如 “冷战” ( cold war)、“反恐战争” （war on terror）、“抗癌战争”

（war on cancer）和 “应对全球变暖的战争”（war on global warming），这样的框定性隐喻 可以激发全国以及社会的团结和超越意识形态的分歧。 ${ }^{[15]}$ 而小布什政府在建构其反恐政策使 
通过使用 “反恐战争”（war on terror）的隐喻, 成功地把 “反恐” 框定为战争, 激发起了美 国民众对于恐怖主义的憎恶情绪，并使其可以通过战争行为来应对恐怖主义。

\section{3. 政治话语中隐喻运用的案例阐释: 对克林顿政府与小布什政府气候变化政策建构的分 析}

在政治实践和政策制定中，类似隐喻这样的话语策略的使用无疑可以增加政治话语或话语的 分量和说服力, 因此在政治话语中隐喻得到了较为广泛的使用。例如, 克林顿政府在对气候 变化制定过程中也通过隐喻策略的使用来达到预期的效果和政治目的一一强化美国民众对 于气候变化问题的关注度, 使气候变化问题成为其政府的重要议题之一。在京都会议之前, 克林顿清楚地感觉到, 在他进行任何严肃的行动之前, 他必须使更多的美国人关注气候变化 问题。1997 年 7 月 24 日，在白宫举行的气候变化会议上，他与政府的首席环保主义者副总 统戈尔展开了一场激发公众更强意识的宣传。总统讲到, “我们看到火车来了, 但大多数美 国人在日常生活中不能听到鸣笛声。”在此, 克林顿总统使用了隐喻来框定问题的严重性并 以此唤起民众的意识。通过 “我们看到火车来了” 说明气候变化是一个已经迫在眉睫的紧迫 问题，而 “大多数美国人在日常生活中不能听到鸣笛声” 则生动地表现出大多数美国民众还 没有意识到气候变化问题的严重性。通过隐喻的使用，使普通民众可以依靠日常生活中常见 的火车来理解气候变化这个他们不熟悉的事物，从而达到建构民众对于气候变化的认知。而 在 1997 年谈到《京都议定书》时, 克林顿总统指出, “当全世界的国家在 12 月齐聚京都参 加联合国气候会议时, 我们所有的参加者, 无论发达国家还是发展中国家，必须抓住机遇， 让温室气体排放的时钟倒转（turn back the clock），这样我们才能留给我们的孩子们一个健 康的星球”。克林顿通过隐喻的使用, 使听众产生 “时钟” 的意象和紧迫感, 起到了对于听 众在气候变化问题上的警醒作用。

而与克林顿政府相反，其继任者小布什政府采取弱化气候变化问题的政策，竭力降低美国民 众对气候变化问题的关注程度和忧虑程度, 并在任期伊始就退出克林顿政府签署的《京都议 定书》。尽管小布什政府的气候变化政策与前任相比有很大的改变, 但在其相关的政治话语 中同样也使用了隐喻的话语策略来建构其政策。例如，小布什政府的美国气候问题首席谈判 代表沃斯在解释美国退出《京都议定书》时讲到，“我们认为有（比《京都议定书》）更好 的方法, 我们不需要跳下悬崖。”他把接受《京都议定书》比喻为 “跳下悬崖”。通过这个 隐喻，他把接受《京都议定书》框定为 “使美国经济背负致命的负担，造成对美国的致命性 影响”。这个隐喻的使用, 强化了小布什政府对于《京都议定书》的否定性框定, 无疑会增 加美国公众对《京都议定书》的负面印象，也会使小布什政府退出《京都议定书》的行为得 到一部分美国公众的支持，在一定程度上可以缓解小布什政府因此行为而受到的国内压力。 而在 2005 年 12 月举行的缔约方第 11 次谈判中, 美国代表团团长多布里扬斯基强调技术在气 候变化应对中的主导作用。他讲到, “八国峰会的成果证明国际范围内对于既有利于民众又 有利于环境的行动的支持。技术是通用货币（common currency）。建立能够支持多种方式的 伙伴关系是最好的出路” 。通过把 “技术” 比喻为 “通用货币” ，多布里扬斯基直接把技术 的使用与经济前景联系起来。基于美国人所熟知的浐语 “金钱使世界运转起来” （Money makes the world go round），这个隐喻的使用意在使人们意识到在气候变化的语境中，技术也 具有如此强大的作用和效果。在其他气候变化相关的场合下，隐喻也被较为频繁地使用。例 如, 美国首席谈判代表沃森在缔约方第 11 次谈判时讲到, “技术是可以把那些经济和环境目 标结合起来的粘合剂（glue）”。把 “技术” 比作 “粘合剂”赋予了技术一种统一（unifying） 和连接 (connecting) 的功能, 使它类似于国家间分歧性目标的连接纽带。 ${ }^{[16]}$ 而另一个使用频繁 的隐喻就是把技术作为 “解开气候变化之钥匙”（the key to climate change）。虽然 “key” 这个词现在更多已被作为一个普通意义的词来使用，但它所蕴含的隐喻意义是其他的替代词 语, 如 solution, way, means, approach 等所不具备的。相比之下, “key” 蕴含着更大的有效 性, 尤其是其 “唯一性” 的含义是其他词语所不具备的。这样，通过这个隐喻可以说明技术 
在解决气候变化问题上的独一性和优势所在，从而对其他方法产生了较强的排它性。例如, 2005 年小布什总统在 “亚太伙伴关系”（Asia-Pacific Partnership）建立时讲到，“发展和利 用更洁净和更高效的创新型技术是解决我们面临的气候挑战的关键所在”。总之，隐喻的使 用在小布什政府把技术框定为气候变化解决方案中起到了一定的作用。

\section{4. 结语}

隐喻在政治话语中的大量使用体现了隐喻对人们的认知具有重要影响作用, 也体现了隐喻所 具有的社会建构和政策建构功能。因此, 对隐喻的认识也要从其传统的修辞修饰功能转向其 所具有的认知功能和社会现实及政策建构功能上, 这样的转向将有助于对于人类认知和社会 事实的本质产生更加深入的理解，同时也将更好地挖掘出隐喻所具有的社会价值。

\section{References}

[1]. Michael P. Marks. Metaphors in International Relations Theory, New York, Palgrave Macmillan, 2011, p.10.

[2]. American Heritage Dictionary of the English Language (3rd Edition), Boston, MA: Houghton Mifflin, 1992.

[3]. G. Lakoff. Women, Fire and Dangerous Things. Chicago, IL: Chicago University Press, 1987.

[4]. Rainer Hülsen, Trans. Wang Zhiqiang. On Metaphor's Function of Social Construction: The EU Expansion as An Example. International Review, Vol. 4 (2008) No.2, p.17.

[5]. G. Lakoff and M. Johnson. Metaphors We Live by. Chicago: The University of Chicago Press, 1980.

[6]. Stephen J. Heidt. Presidential Rhetoric, Metaphor, and the Emergence of the Democracy Promotion Industry. Southern Communication Journal, Vol.78 (2013) No.3, p.235.

[7]. Teun A. Van Dijk. Principles of Critical Discourse Analysis. Discourse \& Society, Vol.4 (1993) No.2, p.249-283.

[8]. N. Fairclough. Language and Power. London: Longman, 1989, p.36-37.

[9]. Paul Ricouer. The Rule of Metaphor: Multi-Disciplinary Studies in the Creation of Meaning in Language, Toronto and Buffalo: University Toronto Press, 1978, p.132.

[10]. P. H. Thibodeau and L. Boroditsy. Metaphors We Think with: The Role of Metaphor, Plos One, Vol.6 (2011) No.2,

http://www.plosone.org/article/info\%3Adoi\%2F10.1371\%2Fjournal.pone.0016782.

[11]. N. Fairclough. Discourse and Social Change. Cambridge: Polity Press, 1992, p.194.

[12]. Mark J. Landau and Lucas A. Keefer. This Is Like That: Metaphors in Public Discourse Shape Attitudes. Social and Personality Psychology Compass, Vol.8 (2014) No.8, p.463.

[13]. B. Nerlich and N. Koteyko. Compounds, Creativity and Complexity in Climate Change Communication: The Case of 'Carbon Indulgences'. Global Environmental Change, Vol.19 (2009) No.3, p.345-353.

[14]. P. H. Thibodeau and L. Boroditsy. Metaphors We Think with: The Role of Metaphor in Reasoning. http://dx.doi.org/10.1371/journal.pone.0016782.

[15]. E. Nepstad. The Continuing Relevance of Coser's Theory of Conflict. Sociological Forum, Vol.20 (2005) No.2, p.335-337.

[16]. Peter Schniering. U. S. Climate Policy and Technology. Baden-Baden: Nomos, 2008, p.129. 